\title{
Saline infusion sonohysterography for uterine cavity evaluation in women with abnormal uterine bleeding
}

\author{
Mohamed Abd El-Sattar ${ }^{1}$, Ahmed M. Abbas ${ }^{2}$, Mohamed K. Tawfik ${ }^{2}$, Mahmoud A. Fouad ${ }^{1}$
}

${ }^{1}$ Department of Obstetrics and Gynecology, Faculty of Medicine, Al-Azhar University, Assiut, Egypt

${ }^{2}$ Department of Obstetrics and Gynecology, Faculty of Medicine, Assiut University, Assiut, Egypt

Received: 20 March 2016

Accepted: 23 April 2016

\section{*Correspondence:}

Dr. Ahmed M. Abbas,

E-mail: bmr90@hotmail.com

Copyright: () the author(s), publisher and licensee Medip Academy. This is an open-access article distributed under the terms of the Creative Commons Attribution Non-Commercial License, which permits unrestricted non-commercial use, distribution, and reproduction in any medium, provided the original work is properly cited.

\begin{abstract}
Background: The aim of this study was to compare the diagnostic effectiveness of saline infusion sonohysterography (SIS) with hysteroscopy, as a gold standard diagnostic method, in detecting endometrial pathology in premenopausal women with abnormal uterine bleeding.

Methods: A prospective study was conducted at Al-Azhar University hospital, Assiut, Egypt. Fifty patients were recruited from the gynecological outpatient clinic complaining of abnormal uterine bleeding. They were evaluated by SIS and compared with hysteroscopic examination for each.

Results: The mean age of patients was $45.56 \pm 3.48$ years. All the intracavitary lesions; endometrial polyps, submucous fibroids and hyperplastic endometrium were equally detected by both methods. Diagnostic accuracy of SIS in comparison with hysteroscopy was $76 \%$ with sensitivity $80 \%$, specificity $72 \%$.

Conclusions: SIS is a reliable and accurate method for evaluation of the uterine cavity. It can be a good alternative technique for the evaluation of uterine cavity abnormalities where hysteroscopy is not available.
\end{abstract}

Keywords: Abnormal uterine bleeding, Saline infusion sonohysterography, Hysteroscopy

\section{INTRODUCTION}

Abnormal uterine bleeding (AUB) is one of the main reasons for women to consult a gynecologist. Approximately $33 \%$ of all admissions to gynecology clinics and $69 \%$ among postmenopausal women are due to abnormal bleeding. ${ }^{1}$ Confirming the diagnosis and planning the treatment of abnormal uterine bleeding are very important in clinical practice. It is essential to distinguish organic causes from dysfunctional causes. Although dysfunctional causes require medical treatment, organic causes such as endometrial polyp, uterine fibroids, endometrial hyperplasia and endometrial cancer may require surgical treatment. ${ }^{2}$

Transvaginal ultrasonography (TVS) is highly applicable, non-invasive and preferred initially in the evaluation of women with AUB. ${ }^{3}$ However, the accuracy of TVS is limited in the diagnosis of focal endometrial lesions. This can be overcome by saline infusion sonohysterography (SIS), which can be performed easily and rapidly and is well tolerated by patients. ${ }^{4,5}$ Hysteroscopy is an effective procedure but more expensive than SIS. Direct visualization of the uterine cavity is possible by HS but it does not give any information about myometrium and adnexa.

Previous study by our group revealed that the sensitivity and specificity of SIS in diagnosis of intracavitary lesions in patients with infertility were $84.3 \%$ and $75 \%$ respectively. ${ }^{6}$ Hysteroscopy was superior on SIS in the diagnosis of intrauterine adhesions and Mullerian anomalies in a cohort of 104 infertile women. ${ }^{6}$

The aim of the current study was to compare the diagnostic accuracy of SIS with hysteroscopy as a gold 
standard for detecting intracavitary abnormalities in women with AUB.

\section{METHODS}

This was a prospective study included 50 premenopausal women, more than 40 years old, with abnormal uterine bleeding attending the outpatient gynecology clinic, Al Azhar University Hospital, Egypt over a period of one year between November 2014 and October 2015. All patients were invited to participate in this study after taking an informed consent. The study was approved by the Ethical Review Board of Al Azhar faculty of medicine.

Abnormal uterine bleeding was defined as any excessive, prolonged or irregular uterine bleeding outside the patient's menstrual cyclic pattern. We excluded patients with previous history of cervical surgery, recent pelvic infection and those who are suspected to be pregnant. Patients who refused to participate in the study were also excluded from the study.

A detailed menstrual history, systemic and general examination was done. Examination of the uterine cavity by SIS and Hysteroscopy was performed for all patients, between day 5 and day 10 postmenstrual, sequentially.

SIS was performed using a $5.0-\mathrm{MHz}$ vaginal probe, Medison-X6 ultrasound machine, and all women were evaluated by the same sonographer. Bimanual examination of the pelvis was performed firstly for the patient in the lithotomy position. Then, Cusco speculum was inserted in the vagina and disinfection of the cervix and the vagina with povidone-iodine solution. The upper cervical lip was grasped with a tenaculum, a sterile 8-F Foley catheter (length, $30 \mathrm{~cm}$; diameter, $2.7 \mathrm{~mm}$ ) was inserted through the cervical os into the endometrial cavity until it reached the fundus and its balloon was filled with $2 \mathrm{ml}$ of sterile saline solution.

After that, the tenaculum and speculum were removed and the ultrasound probe introduced into the vagina. A $50-\mathrm{mL}$ syringe containing physiologic sterile saline $(0.9 \% \mathrm{NaCl})$ used for saline instillation and distension of the uterine cavity with the saline. The measurements of the endometrium were performed at the thickest part in the longitudinal view of the uterus. The uterine cavity contours were inspected for irregularities and suspicious intracavitary lesions were recorded.

Hysteroscopy was carried out in the operating theatre using a rigid microhysteroscope with a $4 \mathrm{~mm}$ diagnostic sheath under general anesthesia. Glycine was used as a distention medium. A maximum intrauterine pressure of $100 \mathrm{~mm} \mathrm{Hg}$ was allowed. The cavity was evaluated visually, with both tubal ostia being noted and the endometrial appearances documented. Another investigator, who was blinded to the results of SIS evaluation, performed the hysteroscopic examination.
This was considered the gold standard for the diagnosis of any intracavitary lesions

The data were collected and entered in to Microsoft excel to be analysed using the Statistical Package for Social Science (SPSS Inc., Chicago, version 21). Qualitative variables were expressed as percentages and compared by Fisher's exact test. Level of significance "P" value was evaluated, where $\mathrm{P}<0.05$ is considered of significant value. Measures of sensitivity, specificity, positive and negative predictive values for SIS were based on the hysteroscopy results.

\section{RESULTS}

The mean age of study participants was $45.56 \pm 3.48$ years. Menorrhagia was the most typical presentation found in $31(62 \%)$ women, while metrorrhagia was present in 16 (32\%) women and 3 cases suffered from polymenorrhea.

Table 1: Correlation between SIS and Hysteroscopic findings in the study participants.

\begin{tabular}{|llll|}
\hline & \multicolumn{3}{c|}{ Study participants $(\mathbf{n = 1 0 4 )}$} \\
\hline Normal & SIS & Hysteroscopy & P-value \\
\hline Endometrial polyp & 23(46\%) & $24(48 \%)$ & 0.236 \\
\hline Submucous fibroid & $8(16 \%)$ & $7(14 \%)$ & 0.359 \\
\hline $\begin{array}{l}\text { Hyperplastic } \\
\text { endometrium }\end{array}$ & $4(8 \%)$ & $2(4 \%)$ & 0.742 \\
\hline
\end{tabular}

SIS: Saline infusion sonohysterography; Fisher's exact test was used to compare the difference in proportions.

Abnormal findings were seen in 26 patients (52\%), while the remaining 24 patients showed normal endometrium (Table 1). Endometrial polyps were the most frequent detected uterine pathology by SIS and Hysteroscopy. Endometrial polyps were diagnosed in 17 patients (34\%) by hysteroscopy. SIS missed the diagnosis of 2 cases and hysteroscopy confirmed the presence of an endometrial polyp. Submucous fibroid was diagnosed in 8 women. One of them was not confirmed with the hysteroscopic examination of the uterine cavity (false positive results). Sensitivity and specificity of SIS in detecting intracavitary lesions were $80 \%$ and $72 \%$ respectively. The overall accuracy was $76 \%$.

\section{DISCUSSION}

There are many tools used in diagnosis of intrauterine pathology, the most frequently used being TVS, SIS, diagnostic hysteroscopy and endometrial sampling, used individually or in combination. The choice of one test over another will depend primarily on its diagnostic accuracy. ${ }^{7}$

AUB is a very common symptom in women of all ages. For patients with AUB, TVS is performed as an initial investigation. In cases where TVS demonstrates an abnormal endometrial thickness or if TVS is suboptimal, 
SIS or hysteroscopy is performed. SIS is also recommended in patients with normal TVS who are unresponsive to medical management. Evaluation of AUB is of special importance in postmenopausal women, because of the high prevalence of endometrial cancer in this women. ${ }^{8}$

Diagnostic hysteroscopy has generally been accepted as the gold standard for evaluation of the uterine cavity. It is an invasive procedure, which is associated with discomfort for the patients and sometimes vasovagal attack. It can be performed in the office setting or as a day-case procedure. ${ }^{9}$ But, it is a more expensive and more invasive than ultrasonography. It allows for direct visualization of the endometrial cavity, with an accurate assessment of intracavitary lesions, and thus accurate removal of lesions such as polyps and submucosal fibroids. ${ }^{8}$ An alternative to diagnostic hysteroscopy could be SIS, which is used to evaluate the uterine cavity after application of fluid medium.

Both SIS and hysteroscopy have excellent diagnostic accuracy in diagnosing submucous fibroids, polyps, and endometrial hyperplasia. ${ }^{9}$ Our results agreed with them as we found no statistically significant differences between SIS and hysteroscopy in percentage of diagnosed intracavitary lesions.

In our study the Sensitivity, Specificity, Positive and negative predictive values of SIS in patients with AUB was $(80,72 \%, 74.07$ and $78.26 \%$ respectively). These results were analysed using the hysteroscopy as a gold standard method for diagnosis of intracavitary lesions.

Glanc et al reported a higher performance of SIS as an investigation tool in premenopausal women with AUB, with a sensitivity and specificity of $95 \%$ and $88 \%$, respectively. ${ }^{10}$ Our findings also were less than those of Dueholm et al, who reported in their study that the overall sensitivity of SIS was $83 \%$, specificity $90 \%$, positive predictive value $85 \%$ and negative predictive value $89 \% .^{4}$

Hysteroscopy cannot be used as a first-line test in all women with AUB, because it is invasive and expensive. However, SIS when combined with TVS can be a reliable, first-line investigation procedure in women with AUB as it is rapid, cost-effective, and is relatively safe. ${ }^{2}$

In summary, SIS is rapid, safe, highly effective, and a less invasive method in comparison to hysteroscopy. SIS can be used as a first-line diagnostic approach in patients with AUB.
Funding: No funding sources

Conflict of interest: None declared

Ethical approval: The study was approved by the Institutional Ethics Committee

\section{REFERENCES}

1. Kelekci S, Kaya E, Alan M, Alan Y, Bilge U, Mollamahmutoglu L. Comparison of transvaginal sonography, saline infusion sonography, and office HS in repro-ductive-aged women with or without abnormal uterine bleeding. Fertil Steril. 2005;84:682-6.

2. Soguktas S, Cogendez E, Kayatas SE, Asoglu MR, Selcuk S, Ertekin A. Comparison of saline infusion sonohysterography and hysteroscopy in diagnosis of premenopausal women with abnormal uterine bleeding. Eur J Obstet Gynecol Reprod Biol. 2012;161(1):66-70.

3. Dreisler E, Sorensen SS, Ibsen PH, Lose G. Value of endometrial thickness measurement for diagnosing focal intrauterine pathology in women without abnormal uterine bleeding. Ultrasound Obstet Gynecol. 2009;33:344-8.

4. Dueholm M, Forman A, Jensen ML, Laursen H, Kracht P. Transvaginal sonogra-phy combined with saline contrast sonohysterography in evaluating the uterine cavity in premenopausal patients with abnormal uterine bleeding. Ultrasound Obstet Gynecol. 2009;18:54-61.

5. Mathew M, Gowri V, Rizvi SG. Saline infusion sonohysterography-an effective tool for evaluation of the endometrial cavity in women with abnormal uterine bleeding. Acta Obstet Gynecol Scand. 2010;89:140-2.

6. Abbas AM, Tawfik MK, Tammam AE, Abdellah AH, Mwafy A. The diagnostic value of saline infusion sonohysterography versus hysteroscopy in evaluation of uterine cavity in patients with infertility and recurrent pregnancy loss. Thai J Obstet Gynecol. 2015;23:113-7.

7. Krampl E, Bourne T, Solbakken HH, Istre O. Transvaginal ultrasonography, sonohysterography and operative hysteroscopy for the evaluation of abnormal uterine bleeding. Acta Obstet Gynecol Scand. 2001;80:616-22.

8. Alfhaily F, Ewies A. The first-line investigation of postmenopausal bleeding: Transvaginal ultrasound scanning and endometrial biopsy may be enough. Int J Gynecol Cancer 2009; 19:892-5.

9. Gimpelson RJ, Whalen TR. Hysteroscopy as gold standard for evaluation of abnormal uterine bleeding. Am J Obstet Gynecol. 1995;173:1637-8.

10. Glanc P, Betel C, LevToaff A. Sonohysterography: Technique and clinical applications. Ultrasound Clin. 2008;3:427-31.

Cite this article as: El-Sattar MA, Abbas AM, Tawfik MK, Fouad MA. Saline infusion sonohysterography for uterine cavity evaluation in women with abnormal uterine bleeding. Int J Reprod Contracept Obstet Gynecol 2016;5:1843-5. 